\title{
A Comparison of the Variable Sampling Interval (VSI) and Variable Sample Size (VSS) Coefficient of Variation Charts
}

\author{
Sok Li Lim', Wai Chung Yeong ${ }^{2}$, Michael Boon Chong Khoo ${ }^{3}$, Xin Ying Chew \\ ${ }^{1}$ Institute of Mathematical Sciences, Faculty of Science, Universiti Malaya \\ 50603 Kuala Lumpur, Malaysia \\ sokli@um.edu.my \\ ${ }^{2}$ Department of Operations and Management Information Systems, Faculty of Business and Accountancy \\ Universiti Malaya, 50603 Kuala Lumpur, Malaysia \\ yeongwc@um.edu.my \\ ${ }^{3}$ School of Mathematical Sciences, Universiti Sains Malaysia \\ 11800 Penang, Malaysia \\ mkbc@usm.my \\ ${ }^{4}$ School of Computer Sciences, Universiti Sains Malaysia \\ 11800 Penang, Malaysia \\ xinying@usm.my
}

\begin{abstract}
An efficient way to perform quality monitoring is important for a lot of industries, particularly in the manufacturing industry. Control charts are one of the important tools to monitor quality. Recently, control charts monitoring the coefficient of variation (CV) are proposed to monitor processes which do not have a constant mean and a standard deviation which changes with the mean. These processes cannot be monitored by standard control charts which monitor the mean and/or standard deviation. The Variable Sampling Interval (VSI) and Variable Sample Size (VSS) CV charts are two recent charts proposed to improve the detection ability of CV charts. This paper compares the performance between these two charts in terms of the Average Time to Signal (ATS). The comparison shows that the VSS CV chart shows better performance when either a small sample size is taken or when a small shift in the CV needs to be detected. Alternatively, the VSI CV chart outperforms the VSS CV chart. This paper will enable practitioners to know the conditions where the VSS CV chart outperforms the VSI CV chart, and vice versa. Hence, practitioners will be able to decide whether it is better to monitor their processes with a VSI or VSS scheme.
\end{abstract}

Keywords: Average Time to Signal, Coefficient of Variation, Sample Size, Shift Size, Variable Sampling Interval, Variable Sample Size.

\section{Introduction}

Control charts are useful tools which can be efficiently utilized for process monitoring, especially for manufacturing processes. However, most control charts are designed to monitor changes in the mean and/or standard deviation of the process, which assumes that changes in the mean and/or standard deviation shows that the process is out-of-control. However, there are many in-control processes where the mean is expected to fluctuate and the standard deviation changes with the mean. This frequently happens when we are monitoring some process output which typically changes from time to time. For example, the amount of a certain chemical in a patient's blood varies from patient to patient. Another example where the mean changes is when the contaminants released day to day in the atmosphere by industrial plants are monitored, since the amount of processing can change based on a daily schedule. In such processes, the use of the traditional $\bar{X}$ and $S$ or $R$ charts is dubious. However, if the process standard deviation is a linear function of the process mean, the sample coefficient of variation $(\mathrm{CV})$ can be used to keep track of the process variability and to detect shifts in the process mean or standard deviation due to assignable causes.

The CV is widely used in many different fields. In the field of manufacturing and materials engineering, some quality characteristics related to the physical properties of metal alloys or composite materials often have a standard deviation which is proportional to the population mean. These properties are usually related to the way atoms of a metal diffuse into another. 
Tool cutting life and several properties of sintered materials are typical examples from this setting [1]. It is also adopted in clinical and diagnostic areas, where the $\mathrm{CV}$ is often used as a measurement to determine the amount of certain chemicals in a patient's urine and blood to diagnose the presence of a disease [2 - 4]. In oncology, the CV is used to evaluate the repeatability in bi-dimensional computed tomography measurements [5]. In physiological science, the CV can be applied to assess the homogeneity of bone sample [6]. Monitoring the CV is also important in chemical and biological assay quality control to validate results [7-8]. In investments, the CV is used to measure the volatility in comparison to the expected return. A lower CV indicates a higher expected return with less risk [9]. The CV is also used to measure the stability of dividend yields of the Hang Seng index [10].

The first control chart to monitor the CV was proposed by [11]. However, the chart only considers the current sample and is not efficient in detecting small shifts in the CV. This results in different charts being proposed to improve the efficiency in process monitoring through the CV. For example, [1, 12-13] proposed the Exponentially Weighted Moving Average (EWMA) chart, [14] proposed the synthetic chart, [15] proposed the run rules chart, [16] proposed the side sensitive group runs (SSGR) chart and [17] proposed the run sum chart. These charts result in an improvement compared to the chart proposed by [11].

The CV charts proposed in the preceding paragraph are based on fixed chart parameters, i.e. a fixed sample size is taken according to a fixed sampling interval, and the charting statistics are compared against fixed control limits. However, it was found that adopting adaptive strategies, i.e. where the chart parameters are varied according to the information obtained from the previous sample, brings about a significant improvement on the performance of a control chart. Hence, this paper would like to compare the performance of two adaptive CV charts in the literature, i.e. the Variable Sampling Interval (VSI) and the Variable Sample Size (VSS) CV charts. The performance of these charts are compared in terms of the Average Time to Signal (ATS), which evaluate the average time required until an out-of-control signal is produced. There are two types of ATS, the in-control ATS $\left(\mathrm{ATS}_{0}\right)$ and the out-of-control ATS $\left(\mathrm{ATS}_{1}\right)$. The $\mathrm{ATS}_{0}$ evaluates the false alarm rate, where a smaller $\mathrm{ATS}_{0}$ shows more frequent false alarms, while the $\mathrm{ATS}_{1}$ evaluates the average time the chart takes to identify an out-ofcontrol condition, where a smaller $\mathrm{ATS}_{1}$ shows that the chart is better in detecting abnormalities in the process. When the performances of these charts are compared, the charts are optimized to have the best $\mathrm{ATS}_{1}$ performance, subject to constraints in the $\mathrm{ATS}_{0}$.

Through the comparison in this paper, practitioners will be able to know whether the VSI or VSS charts perform better, thereby facilitating practitioners to identify the appropriate chart to adopt for process monitoring. The paper is organized as follows. The next section reviews the VSI and VSS CV charts. This is followed by Section 3 which compares the performance of these charts. Lastly, concluding remarks are given in Section 4.

\section{Variable Sampling Interval (VSI) and Variable Sample Size (VSS) Coefficient of Variation (CV) Charts}

This section gives a review of the VSI and VSS CV charts. This includes a review on how the charts operate, the formulae to compute the performance measures, and the algorithm to obtain the optimal chart parameters.

\subsection{Variable Sampling Interval (VSI) Coefficient of Variation (CV) Chart}

The VSI CV chart was proposed by [18]. The VSI CV chart operates by varying the sampling interval $(h)$ according to whether the most recent sample $\mathrm{CV}(\hat{\gamma})$ falls in the central or warning region. When $\hat{\gamma}$ falls in the central region, it is unlikely that the process is out-of-control, hence less stringent monitoring is conducted, where a long sampling interval $\left(h_{L}\right)$ is adopted. Conversely, when $\hat{\gamma}$ falls in the warning region, there are some evidence that the process is going out-of-control, hence more stringent monitoring is conducted, where a short sampling interval $\left(h_{s}\right)$ is adopted. Note that $h_{s}$ shows more frequent sampling, while $h_{L}$ shows less frequent sampling. Figure 1 shows the operation of the chart. 


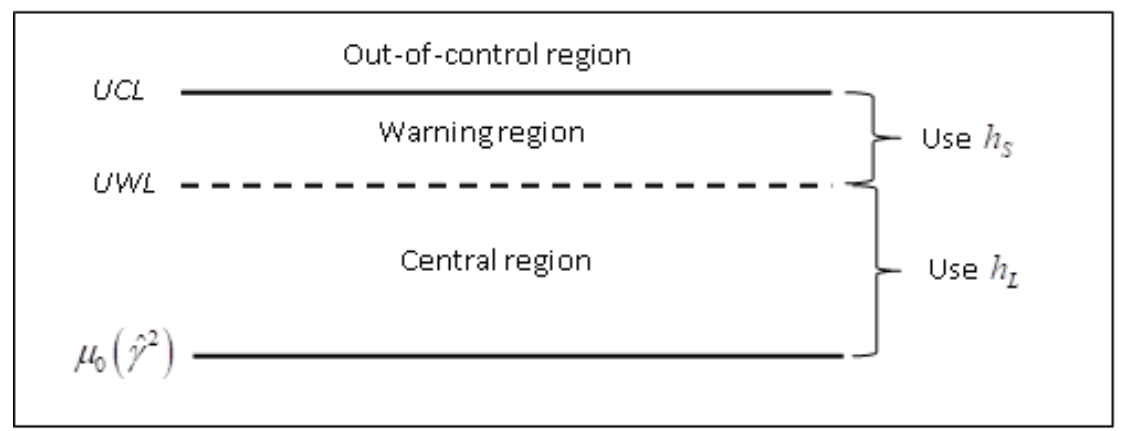

Fig. 1: A graphical view of the VSI CV chart's operation.

The $U C L$ and $U W L$ are the control and warning limits, respectively. The control and warning limits can be calculated as follows:

$$
\begin{aligned}
& U C L=\mu_{0}(\hat{\gamma})+K \sigma_{0}(\hat{\gamma}), \\
& U W L=\mu_{0}(\hat{\gamma})+W \sigma_{0}(\hat{\gamma}),
\end{aligned}
$$

where $K>W>0$, and $\mu_{0}(\hat{\gamma})$ and $\sigma_{0}(\hat{\gamma})$ are the mean and the standard deviation of the sample CV when the process is incontrol. Note that $K$ is the control limit coefficient and $W$ is the warning limit coefficient. $\mu_{0}(\hat{\gamma})$ and $\sigma_{0}(\hat{\gamma})$ are approximated by [19] as follows:

$$
\begin{gathered}
\mu_{0}(\hat{\gamma}) \simeq \gamma_{0}\left[1+\frac{1}{n}\left(\gamma_{0}^{2}-\frac{1}{4}\right)+\frac{1}{n^{2}}\left(3 \gamma_{0}^{4}-\frac{\gamma_{0}^{2}}{4}-\frac{7}{32}\right)+\frac{1}{n^{3}}\left(15 \gamma_{0}^{6}-\frac{3 \gamma_{0}^{4}}{4}-\frac{7 \gamma_{0}^{2}}{32}-\frac{19}{128}\right)\right], \\
\sigma_{0}(\hat{\gamma}) \simeq \gamma_{0}\left[\frac{1}{n}\left(\gamma_{0}^{2}+\frac{1}{2}\right)+\frac{1}{n^{2}}\left(8 \gamma_{0}^{4}+\gamma_{0}^{2}+\frac{3}{8}\right)+\frac{1}{n^{3}}\left(69 \gamma_{0}^{6}+\frac{7 \gamma_{0}^{4}}{2}+\frac{3 \gamma_{0}^{2}}{4}+\frac{3}{16}\right)\right]^{\frac{1}{2}} .
\end{gathered}
$$

The ATS of the VSI CV chart can be evaluated from the following formula [18]:

$$
\operatorname{ATS}=\frac{h_{S} p_{S}+h_{L} p_{L}}{q(1-q)}
$$

where $p_{L}=F_{\hat{\gamma}}(U W L \mid n, \gamma)-F_{\hat{\gamma}}(L W L \mid n, \gamma)$ (with $n$ and $\gamma$ being the sample size and CV, respectively) is the probability of the sample falling in the central region, $p_{S}=F_{\hat{\gamma}}(U C L \mid n, \gamma)-F_{\hat{\gamma}}(L C L \mid n, \gamma)-p_{L}$ is the probability of the sample falling in the warning region and $q=1-p_{S}-p_{L}$ is the probability of the sample falling in the out-of-control region. Note that $F_{\hat{\gamma}}($. is the c.d.f. of $\hat{\gamma}$ given by $F_{\hat{\gamma}}(x \mid n, \gamma)=1-F_{t}\left(\frac{\sqrt{n}}{x} \mid n-1, \frac{\sqrt{n}}{\gamma}\right)$, where $F_{t}(\cdot)$ is the c.d.f. of the non-central $t$ distribution with $n-1$ degrees of freedom and non-centrality parameter $\frac{\sqrt{n}}{\gamma}$. 
The $\mathrm{ATS}_{0}$ and $\mathrm{ATS}_{1}$ is obtained by setting $\gamma=\gamma_{0}$ and $\gamma=\gamma_{1}$, respectively, into Eq. (5), where $\gamma_{0}$ and $\gamma_{1}$ are the incontrol and out-of-control CV, respectively. $\gamma_{1}$ is given by $\gamma_{1}=\tau \gamma_{0}$, where $\tau$ is the magnitude of the shift in the in-control CV.

Five chart parameters need to be determined in order to implement the VSI CV chart, i.e. the sample size $(n)$, the long sampling interval $\left(h_{L}\right)$, the short sampling interval $\left(h_{S}\right)$, the warning limit coefficient $(W)$ and the control limit coefficient $(K)$. $n$ is fixed by the practitioner depending on the availability of resources, while possible combinations of $\left(h_{S}, h_{L}\right)$ were provided by [18]. The value of $K$ is set to obtain a fixed value of $\mathrm{ATS}_{0}$, while $W$ is set to obtain a fixed value of the in-control Average Sampling Interval $\left(\mathrm{ASI}_{0}\right)$, where $\mathrm{ASI}=\frac{h_{S} p_{S}+h_{L} p_{L}}{1-q}$

\subsection{Variable Sample Size (VSS) Coefficient of Variation (CV) Chart}

The VSS CV chart was proposed by [20] and [21]. [20] monitored a transformed function of the CV, while [21] monitored the CV directly. The VSS CV chart by [21] is adopted in this paper. Like the VSI CV chart, more stringent control is adopted when there are signs that the process is going out-of-control. However, unlike the VSI CV chart, the VSS CV chart takes a larger sample size $\left(n_{L}\right)$ when $\hat{\gamma}$ falls in the warning region, while a smaller sample size $\left(n_{S}\right)$ is taken when $\hat{\gamma}$ falls in the central region.

From Eqs. (1) and (2), it can be observed that $U C L$ and $U W L$ are functions of the sample size $(n)$ through the functions $\mu_{0}(\hat{\gamma})$ and $\sigma_{0}(\hat{\gamma})$. Hence, when different sample sizes are adopted, the $U C L$ and $U W L$ will be different. Thus, the VSS CV chart will have two pairs of warning and control limits. The first pair $\left(U C L_{S}, U W L_{S}\right)$ is adopted when the current sample is $n_{S}$, while the second pair $\left(U C L_{L}, U W L_{L}\right)$ is adopted when the current sample is $n_{L}$. The following shows the formulae to compute $U C L_{S}, U C L_{L}, U W L_{S}$ and $U W L_{L}$.

$$
\begin{aligned}
& U C L_{S}=\mu_{0}(\hat{\gamma})+K_{S} \sigma_{0}(\hat{\gamma}), \\
& U C L_{L}=\mu_{0}(\hat{\gamma})+K_{L} \sigma_{0}(\hat{\gamma}), \\
& U W L_{S}=\mu_{0}(\hat{\gamma})+W_{S} \sigma_{0}(\hat{\gamma}), \\
& U W L_{L}=\mu_{0}(\hat{\gamma})+W_{L} \sigma_{0}(\hat{\gamma}),
\end{aligned}
$$

where $W_{S}$ and $W_{L}$ are the warning limit coefficients when the current sample is small and large, respectively, while $K_{S}$ and $K_{L}$ are the corresponding control limit coefficients.

To evaluate the ATS of the VSS CV chart, a Markov chain approach is adopted. By defining the first and second states as the central and warning regions, respectively, while the third state is defined as the out-of-control region, [21] has shown that the transition probability matrix (tpm) $\mathbf{P}$ has the following structure:

$$
\mathbf{P}=\left(\begin{array}{cc}
\mathbf{Q} & \mathbf{r} \\
\mathbf{0}^{\mathbf{T}} & 1
\end{array}\right)=\left(\begin{array}{ccc}
p_{S}\left(n_{S}\right) & p_{L}\left(n_{S}\right) & 1-p_{S}\left(n_{S}\right)-p_{L}\left(n_{S}\right) \\
p_{S}\left(n_{L}\right) & p_{L}\left(n_{L}\right) & 1-p_{S}\left(n_{L}\right)-p_{L}\left(n_{L}\right) \\
0 & 0 & 1
\end{array}\right),
$$

with $p_{S}\left(n_{S}\right), p_{S}\left(n_{L}\right), p_{L}\left(n_{S}\right)$ and $p_{L}\left(n_{L}\right)$ being computed as follows:

$$
p_{S}\left(n_{S}\right)=F_{\hat{\gamma}}\left(U W L_{S} \mid n_{S}, \gamma\right)-F_{\hat{\gamma}}\left(L W L_{S} \mid n_{S}, \gamma\right)
$$




$$
\begin{gathered}
p_{S}\left(n_{L}\right)=F_{\hat{\gamma}}\left(U W L_{L} \mid n_{L}, \gamma\right)-F_{\hat{\gamma}}\left(L W L_{L} \mid n_{L}, \gamma\right), \\
p_{L}\left(n_{S}\right)=F_{\hat{\gamma}}\left(L W L_{S} \mid n_{S}, \gamma\right)-F_{\hat{\gamma}}\left(L C L_{S} \mid n_{S}, \gamma\right)+F_{\hat{\gamma}}\left(U C L_{S} \mid n_{S}, \gamma\right)-F_{\hat{\gamma}}\left(U W L_{S} \mid n_{S}, \gamma\right), \\
p_{L}\left(n_{L}\right)=F_{\hat{\gamma}}\left(L W L_{L} \mid n_{L}, \gamma\right)-F_{\hat{\gamma}}\left(L C L_{L} \mid n_{L}, \gamma\right)+F_{\hat{\gamma}}\left(U C L_{L} \mid n_{L}, \gamma\right)-F_{\hat{\gamma}}\left(U W L_{L} \mid n_{L}, \gamma\right),
\end{gathered}
$$

where $F_{\hat{\gamma}}($.$) is the c.d.f. of \hat{\gamma}$ as defined in Section 2.1. From [21], the ATS can be computed as

$$
\operatorname{ATS}=\mathbf{q}^{\mathrm{T}}(\mathbf{I}-\mathbf{Q})^{-1} \mathbf{1}
$$

where $\mathbf{q}=(0,1)^{\mathrm{T}}$ and $\mathbf{1}=(1,1)^{\mathrm{T}}$. Similar with the VSI CV chart, the $\mathrm{ATS}_{0}$ and $\mathrm{ATS}_{1}$ are obtained by setting $\gamma=\gamma_{0}$ and $\gamma=\gamma_{1}$, respectively, into Eq. (15).

Seven chart parameters need to be determined in order to implement the VSS CV chart, i.e. the sampling interval $(h)$, large sample size $\left(n_{L}\right)$, small sample size $\left(n_{S}\right)$, warning limit coefficients $\left(W_{s}\right.$ and $\left.W_{L}\right)$ and control limit coefficients $\left(K_{S}\right.$ and $\left.K_{L}\right) . h$ is fixed by the practitioner depending on the availability of resources. To optimize the performance of the VSS CV chart, the combinations $\left(n_{S}, n_{L}, W_{S}, W_{L}, K_{S}, K_{L}\right)$ which minimizes $\mathrm{ATS}_{1}$, subject to constraints in $\mathrm{ATS}_{0}$ and the in-control Average Sample Size $\left(\mathrm{ASS}_{0}\right)$, will be obtained. From [21], the ASS can be computed as

$$
\mathrm{ASS}=\left(\begin{array}{lll}
n_{S} & n_{L} & n_{L}
\end{array}\right) \mathbf{R}^{-1}\left(\begin{array}{l}
0 \\
1 \\
0
\end{array}\right),
$$

where $\mathbf{R}=\left(\begin{array}{ccc}p_{S}\left(n_{S}\right)-1 & p_{S}\left(n_{L}\right) & 0 \\ 1 & 1 & 1 \\ 1-p_{S}\left(n_{S}\right)-p_{L}\left(n_{S}\right) & 1-p_{S}\left(n_{L}\right)-p_{L}\left(n_{L}\right) & -1\end{array}\right)$.

\section{Performance Comparison}

In this section, we compare the $\mathrm{ATS}_{1}$ performance between the VSI and VSS CV charts. Note that the smaller the ATS the faster the chart can detect an out-of-control condition. To ensure a fair comparison between the charts, both charts need to be designed to satisfy the constraints $\mathrm{ATS}_{0}=370.4, \mathrm{ASI}_{0}=h=1$ and $\mathrm{ASS}_{0}=n$, where this paper considers $n \in\{5,7,10,15\}$ to study the impact of different sample sizes on the performance of the charts. Furthermore, this paper also considers different values of shift sizes, where $\tau \in\{1.10,1.20,1.50,2.00\}$. Note that $\tau$ shows the magnitude of shift in the CV we are interested to detect, where small values of $\tau$ shows that we are interested in detecting a small shift in the CV, while large values of $\tau$ shows that we are interested in detecting a large shift. The in-control CV, $\gamma_{0}$, is set as 0.05 . Table 1 shows the ATS performances of the charts. 
Table 1: $\mathrm{ATS}_{1}$ of the VSI and VSS CV charts, for $n \in\{5,7,10,15\}, \tau \in\{1.10,1.20,1.50,2.00\}$ and $\gamma_{0}=0.05$.

\begin{tabular}{|c|c|c|}
\hline \multicolumn{3}{|c|}{$n=5$} \\
\hline$\tau$ & VSI CV & VSS CV \\
\hline 1.10 & 103.10 & 98.80 \\
\hline 1.20 & 38.90 & 27.20 \\
\hline 1.50 & 6.10 & 5.20 \\
\hline 2.00 & 1.50 & 2.30 \\
\hline \multicolumn{3}{|c|}{$n=7$} \\
\hline$\tau$ & VSI CV & VSS CV \\
\hline 1.10 & 87.60 & 84.50 \\
\hline 1.20 & 29.60 & 20.70 \\
\hline 1.50 & 4.00 & 4.00 \\
\hline 2.00 & 1.00 & 1.80 \\
\hline \multicolumn{3}{|c|}{$n=10$} \\
\hline$\tau$ & VSI CV & VSS CV \\
\hline 1.10 & 73.50 & 72.40 \\
\hline 1.20 & 21.70 & 16.20 \\
\hline 1.50 & 2.40 & 3.10 \\
\hline 2.00 & 0.60 & 1.50 \\
\hline \multicolumn{3}{|c|}{$n=15$} \\
\hline$\tau$ & VSI CV & VSS CV \\
\hline 1.10 & 59.70 & 62.80 \\
\hline 1.20 & 14.80 & 13.20 \\
\hline 1.50 & 1.40 & 2.40 \\
\hline 2.00 & 0.40 & 1.20 \\
\hline \multicolumn{3}{|c|}{} \\
\hline
\end{tabular}

From Table 1, the VSS CV chart outperforms the VSICV chart for the following values of $\tau$ and $n: \tau \in\{1.10,1.20,1.50\}$ when $n=5, \tau \in\{1.10,1.20\}$ when $n \in\{7,10\}$ and $\tau=1.20$ when $n=15$. However, the outperformance of the VSS CV chart reduces as the sample size becomes larger. For $\tau=1.50$ and $n=7$, the VSS and VSI CV charts show the same performance. Meanwhile, for $\tau=2.00$ when $n \in\{5,7\}, \tau \in\{1.50,2.00\}$ when $n \in\{10,15\}$ and $\tau=1.10$ when $n=15$, the VSI CV chart is superior.

Hence, the comparison shows that the VSS CV chart generally outperforms the VSI CV chart for small sample sizes and/or small shift sizes. As the sample size increases, there are fewer advantages of using the VSS CV chart compared to the VSI CV chart. Furthermore, a larger shift size also shows fewer advantages of using the VSS CV chart. Thus, generally, practitioners are recommended to adopt the VSS CV chart when either: (i) there are constraints in resources such that only a small sample size can be taken or (ii) small shifts in the CV needs to be detected.

\section{Conclusion}

This paper compares the performance of the Variable Sampling Interval (VSI) and Variable Sample Size (VSS) Coefficient of Variation (CV) charts. The VSI CV chart varies the sampling interval by taking more frequent samples when there are signs that the process is out-of-control, while the VSS CV chart varies the sample size by taking a larger sample when there are signs that the process is out-of-control. Comparisons show that the VSS CV chart outperforms the VSI CV chart when either the sample size or the shift size is small. Meanwhile, when either the sample size or shift size is large, the VSI CV chart outperforms the VSS CV chart. Hence, the adoption of the VSS scheme is recommended to monitor changes in the $\mathrm{CV}$ when either constraints in the resources results in having to take a small sample or when small changes in the $\mathrm{CV}$ 
need to be detected. Alternatively, if either a large sample can be taken or when only a large change in the CV needs to be detected, the VSI scheme is a better option.

\section{Acknowledgements}

This research is supported by RU Grant - Fakulti Program, grant number RF009B-2018.

\section{References}

[1] P. Castagliola, G. Celano and S. Psarakis, "Monitoring the coefficient of variation using EWMA charts," Journal of Quality Technology, vol. 43, no. 3, pp. 249-265, 2011.

[2] F. S. Apple, M. M. Murakami, L. A. Pearce and C. A. Herzog, "Predictive value of cardiac troponin I and T for subsequent death in end-stage renal disease," Circulation, vol. 106, no. 23, pp. 2941-2945, 2002.

[3] S. J. Chadban, E. M. Briganti, P. G. Kerr, D. W. Dunstan, T. A. Welborn, P. Z. Zimmet and R. C. Atkins, "Prevalence of kidney damage in Australian adults: The AusDiab kidney study," Journal of the American Society of Nephrology, vol. 14, pp. S131-S138, 2003.

[4] L. Tian, "Inferences on the common coefficient of variation," Statistics in Medicine, vol. 24, no. 14, pp. 2213-2220, 2005.

[5] L. H. Schwartz, M. S. Ginsberg, D. DeCorato, L. N. Rothenberg, S. Einstein, P. Kijewski and D. M. Panicek, "Evaluation of tumor measurements in oncology: use of film-based and electronic techniques," Journal of Clinical Oncology, vol. 18, no. 10, pp. 2179-2184, 2000.

[6] A. J. Hamer, J. R. Strachan, M. M. Black, C. Ibbotson and R. A. Elson, "A new method of comparative bone strength measurement," Journal of Medical Engineering \& Technology, vol. 19, no. 1, pp. 1-5, 1995.

[7] G. F. Reed, F. Lynn and B. D. Meade, "Use of coefficient of variation in assessing variability of quantitative assays," Clinical and Diagnostic Laboratory Immunology, vol. 9, no. 6, pp. 1235-1239, 2002.

[8] C. Seyler, T. B. Hofstetter, K. Hungerbühler, "Life cycle inventory for thermal treatment of waste solvent from chemical industry: A multi-input allocation model," Journal of Cleaner Production, vol. 13, no. 13, pp. 1211-1224, 2005.

[9] J. D. Curto and J. C. Pinto, "The coefficient of variation asymptotic distribution in the case of non-iid random variables," Journal of Applied Statistics, vol. 36, no. 1, pp. 21-32, 2009.

[10] W. K. Pang, B. W. Yu, M. D. Troutt and H. H. Shui, "A simulation-based approach to the study of coefficient of variation of dividend yields," European Journal of Operational Research, vol. 189, no. 2, pp. 559-569, 2008.

[11] C. W. Kang, M. S. Lee, Y. J. Seong and D. M. Hawkins, "A control chart for the coefficient of variation," Journal of Quality Technology, vol. 39, no. 2, pp. 151-158, 2007.

[12] E. P. Hong, C. W. Kang, J. W. Baek and H. W. Kang, "Development of CV control chart using EWMA technique," Journal of Society of Korea Industrial and Systems Engineering, vol. 31, no. 4, pp. 114-120, 2008.

[13] J. Zhang, Z. Li, B. Chen and Z. Wang, "A new exponentially weighted moving average control chart for monitoring the coefficient of variation," Computers \& Industrial Engineering, vol. 78, pp. 205-212, 2014.

[14] M. E. Calzada, and S. M. Scariano, "A synthetic control chart for the coefficient of variation," Journal of Statistical Computation and Simulation, vol. 83, no. 5, pp. 853-867, 2013.

[15] P. Castagliola, A. Achouri, H. Taleb, G. Celano and S. Psarakis, "Monitoring the coefficient of variation using control charts with run rules," Quality Technology \& Quantitative Management, vol. 10, no. 1, pp. 75-94, 2013.

[16] H. W. You, M. B. C. Khoo, P. Castagliola and A. Haq, "Monitoring the coefficient of variation using the side sensitive group runs chart," Quality and Reliability Engineering International, vol. 32, no. 5, pp. 1913-1927, 2016.

[17] W. L. Teoh, M. B. C. Khoo, P. Castagliola, W. C. Yeong and S. Y. Teh, "A run sum control chart for monitoring the coefficient of variation,” European Journal of Operational Research, vol. 257, no. 1, pp. 144-158, 2017.

[18] P. Castagliola, A. Achouri, H. Taleb, G. Celano and S. Psarakis, "Monitoring the coefficient of variation using a variable sampling interval control chart," Quality and Reliability Engineering International, vol. 29, no. 8, pp. 11351149, 2013.

[19] W. Reh and B. Scheffler, "Significance tests and confidence intervals for coefficients of variation," Computational Statistics \& Data Analysis, vol. 22, no. 4, pp. 449-452, 1996.

[20] P. Castagliola, A. Achouri, H. Taleb, G. Celano and S. Psarakis, "Monitoring the coefficient of variation using a variable sample size control chart," International Journal of Advanced Manufacturing Technology, vol. 80, no. 9-12, pp. 1561-1576, 2015. 
[21] W. C. Yeong, M. B. C. Khoo, S. L. Lim, M. H. Lee, "A direct procedure for monitoring the coefficient of variation using a variable sample size scheme," Communications in Statistics - Simulation and Computation, vol. 46, no. 6, pp. 4210-4225, 2017. 